\title{
Sur une Valeur propre d'un Operateur
}

\section{T. Ando ${ }^{1}$ et Martin Zerner ${ }^{2}$}

1 Division of Applied Mathematics, Research Institute of Applied Electricity, Hokkaido University, Sapporo 060, Japan

2 Département de Mathématiques, Université de Nice, F-06034 Nice Cedex, France

\begin{abstract}
The studied eigenvalue is the smallest one of the operator:

$$
H_{\mu}=\mu A^{*} A+i \lambda A^{*}\left(A+A^{*}\right) A,
$$

in the orthogonal complement of the vacuum where $A^{*}$ and $A$ are the creation and annihilation operators. Call this eigenvalue $E(\mu)$ as attention is focused on the dependence on $\mu$. This eigenvalue exists and is a positive number for positive values of $\mu$. Using the fact that the inverse of $H_{\mu}$ is a positive operator, it is proved that $E$ extends to a positive, increasing, analytic function on the whole real line. In particular, $E(0) \neq 0$, contrary to what might have been expected from the fact that $A^{*}\left(A+A^{*}\right) A$ is formally self-adjoint.
\end{abstract}

\section{Position du problème et résultats}

Les spécialistes de la physique des hautes énergies ont été amenés à utiliser l'opérateur:

$$
H_{\lambda, \mu}=\mu A^{*} A+i \lambda A^{*}\left(A+A^{*}\right) A,
$$

où $A$ désigne l'opérateur d'annihilation et donc $A^{*}$ le créateur, que son nom soit loué. (L'étoile désigne l'adjoint, une définition mathématique précise de ces opérateurs sera donnée un peu plus loin.)

Cet opérateur intervient comme hamiltonien de la mécanique quantique des reggeons. Dans cette théorie, c'est l'hamiltonien le plus simple qui puisse décrire un champ avec interaction et il est plausible qu'il décrit l'interaction dominante entre deux hadrons.

Dans ce qui précède, le mot «simple» ne s'applique qu'à l'expression algébrique de l'opérateur. Si on examine la famille un peu plus générale,

$$
H_{\lambda, \mu, v}=v A^{* 2} A^{2}+\mu A^{*} A+i \lambda A^{*}\left(A+A^{*}\right) A,
$$

on voit que pour $v$ non nul il s'agit d'une perturbation compacte d'un opérateur défini positif possédant un inverse compact. On sait que cette circonstance peut faciliter grandement son étude. La situation est radicalement différente avec $H_{\lambda, \mu}$. 
D'abord un problème se pose a priori, celui de la définition de son domaine. Ce problème a été résolu pour $\mu$ différent de zéro: les domaines «maximal»et «minimal» sont les mêmes (ce résultat, dû à Intissar [3] est explicité un peu plus loin). Mais si l'on prend deux à deux ce domaine, celui de $A^{*} A$, et un domaine qui paraisse raisonnable pour $A^{*}\left(A+A^{*}\right) A$, on peut vérifier qu'il n'y a aucune inclusion entre eux. La situation est encore pire pour l'opérateur d'interaction $A^{*}\left(A+A^{*}\right) A$. Ses domaines maximal et minimal ne coïncident pas, il n'est pas autoadjoint sur son domaine maximal. Il a des réalisations autoadjointes mais une des conclusions de cet article est qu'aucune d'entre elles n'intervient dans le passage à la limite $\mu$ tendant vers zéro dans $H_{\lambda, \mu}$. Si tant est qu'on peut définir un opérateur limite, il n'est même pas une extension du minimal!

Les valeurs propres de $H_{\lambda, \mu}$ sont réelles et du signe de $\mu$ (Intissar [3, 4]). On peut en tirer l'impression que quand $\mu$ tend vers zéro, ces valeurs propres tendent vers zéro aussi, c'est du moins ce qui est arrivé à l'un des auteurs avant d'y regarder de plus près. Il n'en est rien, et le principal résultat démontré ici est que si on appelle $E(\mu)$ la plus petite valeur propre non nulle pour $\mu$ positif, $E$ a un prolongement analytique strictement positif pour tout $\mu$ réel.

Il est vraisemblable que la valeur physique de $\mu$ est négative. Le comportement asymptotique de la section efficace aux hautes énergies serait alors en $\sigma^{E(\mu)}, E(\mu)$ donné par ce prolongement analytique ( $\sigma$ est l'énergie). Pour une plus ample discussion du rapport des résultats démontrés ci-dessous avec la théorie physique, on pourra se reporter à Intissar et al. [5].

Pour $\mu=0$, on vérifie que la valeur limite $E(0)$ est bien une valeur propre de $i \lambda A^{*}\left(A+A^{*}\right) A$. Le vecteur propre correspondant n'est évidemment pas dans le domaine d'une réalisation auto-adjointe de $A^{*}\left(A+A^{*}\right) A$ (de telles réalisations existent, on ne le démontre pas ici).

L'absence de toute relation entre les domaines des parties auto-adjointe et antiadjointe de $H_{\lambda, \mu}$ nous a obligé à utiliser une propriété assez spéciale de cet opérateur: dans la représentation de Bargmann, si on se restreint à un demi-axe imaginaire, l'opérateur possède un inverse opérateur intégral à noyau positif.

Ciafaloni et al. [1] ont donné une série de propriétés spectrales de $H_{\lambda, \mu}$. Toutefois, la transcription de leur article dans le langage mathématique usuel pose des problèmes et il semble s'être élevé des désaccords parmi les physiciens quant à la validité de leurs résultats. Intissar a commencé l'étude mathématique de cet opérateur ([3-4]). Nous résumons d'abord certains de ses résultats.

Définition 1. L'espace de Bargmann, que nous noterons $E$, est l'ensemble des fonctions $f$, analytiques sur tout le plan complexe et telles que l'intégrale

$$
\frac{1}{\pi} \int_{\mathbb{C}}|f(x+i y)|^{2} e^{-|x+i y|^{2}} d x d y
$$

converge. La norme de $f$ dans cet espace est la racine carrée de cette intégrale, ce qui en fait un espace de Hilbert. Le produit scalaire correspondant sera noté (., .). Enfin, $E_{0}$ désignera le sous-espace (fermé) de $E$ formé des fonctions qui s'annulent à l'origine.

Notations 1 . On pose pour toute fonction analytique complexe $f$ :

$$
A f(z)=f^{\prime}(z), \quad A^{*} f(z)=z f(z) .
$$


Remarque 1. La notation est justifiée. On a $\left\{f ; f^{\prime} \in E\right\}=\{f ; z f \in E\}$, et si on prend cet ensemble comme domaine commun de $A$ et $A^{*}$, ils sont adjoint l'un et l'autre dans l'espace de Bargmann.

Notations 2. On définit un opérateur dans $E_{0}$ en posant:

$$
H_{\lambda, \mu}=\mu A^{*} A+i \lambda A^{*}\left(A+A^{*}\right) A
$$

avec comme domaine:

$$
D=\left\{f \in E_{0} ; H_{\lambda, \mu} f \in E\right\}
$$

(l'image est bien contenue dans $E_{0}$ ). On notera $H_{\mu}$ au lieu de $H_{1, \mu}$.

Propriétés de $H_{\lambda, \mu}$ :

a) $H_{\lambda, \mu}=\lambda H_{\mu / \lambda}$

b) pour $\mu \neq 0, H_{\lambda, \mu}$ est la fermeture de sa restriction aux polynômes

c) toujours pour $\mu \neq 0, H_{\lambda, \mu}$ a un inverse compact

d) $H_{-\lambda, \mu}=H_{\lambda, \mu}^{*}$

e) les valeurs propres de $H_{\lambda, \mu}$ sont réelles

f) pour $\mu>0, \mathscr{R}\left(H_{\lambda, \mu} f, f\right) \geqq \mu\|f\|^{2}$, pour $\mu<0, \mathscr{R}\left(H_{\lambda, \mu} f, f\right) \leqq \mu\|f\|^{2}$.

Conséquence: les valeurs propres de $H_{\lambda, \mu}$ sont plus grandes que $\mu$ pour $\mu$ positif, plus petites que $\mu$ pour $\mu$ négatif (voir la figure ci-dessous où les parties hachurées du plan ne peuvent pas contenir de valeur propre de $H_{\lambda, \mu}$.)

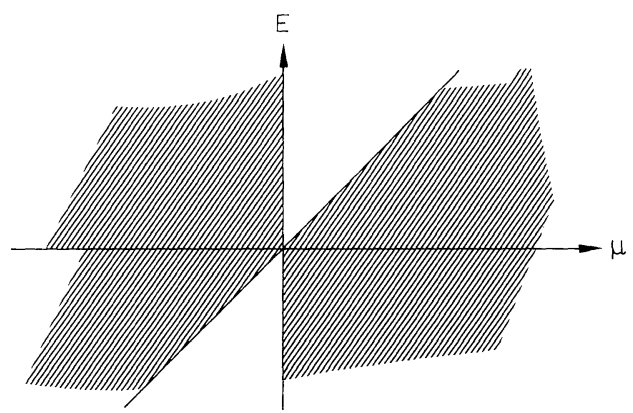

Fig. 1

Les propriétés $b$ et $c$ seront essentielles et la référence [3] est difficile à trouver. Il est donc bon d'indiquer ici une démonstration de ces propriétés.

Notons $H_{\lambda, \mu}^{\min }$ la fermeture de la restriction de $H_{\lambda, \mu}$ aux polynômes (nuls à l'origine puisqu'appartenant à $E_{0}$ ) et $D_{\min }$ son domaine.

Supposons $\mu>0$ pour fixer les idées.

On a pour $u \in D_{\min }$ :

$$
\mathscr{R}\left(H_{\lambda, \mu} u, u\right)=\mu\|A u\|^{2} \geqq \mu\|u\|^{2} .
$$

(Ici et par la suite $\mathscr{R}$ désigne la partie réelle d'un nombre complexe. L'inégalité serait fausse si on n'avait pas éliminé les fonctions constantes en se restreignant à $E_{0}$. Cette inégalité deviendra la propriété $f$ lorsque nous aurons démontré $b$.) 
Ces relations ont deux conséquences:

- $D_{\text {min }}$ est contenu dans le domaine de $A$. En particulier son injection dans $E_{0}$ est compacte (quand on munit $D_{\min }$ de la norme du graphe).

$-H_{\lambda, \mu}^{\min }$ est injectif et son image est fermée.

Nous allons maintenant démontrer que $H_{\lambda, \mu}^{\min }$ est inversible. Au point où nous en sommes il reste pour celà à voir que son image est dense, et pour celà que son adjoint est injectif. On vérifie que cet adjoint n'est autre que $H_{-\lambda, \mu}$. Les seules fonctions nulles à l'origine que $H_{\lambda, \mu}$ annule sont (en écartant le cas particulier $\lambda=0$ )

$$
c \int_{0}^{z} e^{-y^{2} / 2+i \mu y / \lambda} d y
$$

On vérifie «à la main» que ces fonctions n'appartiennent pas à l'espace de Bargmann.

$H_{\lambda, \mu}^{\min }$ est donc inversible et $H_{\lambda, \mu}$ en est une extension injective. Dans cette situation, la seule possibilité est:

$$
H_{\lambda, \mu}=H_{\lambda, \mu}^{\min },
$$

qui est la propriété $b$. La propriété $c$ en résulte puisque nous avons vu que $H_{\lambda, \mu}^{\min }$ était inversible et l'injection de son domaine dans $E_{0}$ compacte.

Proposition 1. Pour $\mu>0, H_{\mu}$ possède au moins une valeur propre. Soit $E(\mu)$ la plus petite de ses valeurs propres. La fonction $E$ se prolonge en une fonction analytique réelle positive et croissante sur $\mathbb{R}$ tout entier.

La démonstration de cette proposition sera l'essential de cet article. Voici quelques indications sur la marche que nous allons suivre. Notons d'abord que le spectre de $H_{\mu}$ est fait de valeurs propres isolées qui sont les inverses des valeurs propres de $H_{\mu}^{-1}$ (d'après la propriété c). C'est le spectre de cet inverse $H_{\mu}^{-1}$ que nous étudierons.

Nous expliciterons d'abord (Sect. 3) cet inverse sous forme d'un opérateur intégral portant sur les restrictions des fonctions au demi-axe imaginaire négatif.

Le point clef est que le noyau de cet opérateur intégral est positif. On dispose donc des généralisations du théorème de Perron-Frobenius. Pour démontrer l'analyticité de $E$, on a besoin de passer dans un autre espace de Banach que $E_{0}$ pour deux raisons:

a) pour $\mu$ négatif l'opérateur intégral obtenu n'opère plus sur $E_{0}$,

b) on a besoin d'une généralisation fine de Perron-Frobenius que nous ne savons pas appliquer dans $E_{0}$.

Le noyau de l'opérateur $H_{\mu}^{-1}$ est analytique par rapport à $\mu$. L'opérateur intégral défini par ce noyau se prolonge en un opérateur compact sur un espace $L^{2}$ avec poids, $y$ compris pour des valeurs négatives de $\mu$ (Sect. 4). Une généralisation du théorème de Perron-Frobenius aux espaces $L^{p}$ (théorème de Jentzsch) permet de démontrer ce qu'on veut sur cette extension, à savoir qu'elle a des valeurs propres non-nulles, que la plus grande d'entre elles en module est positive (donc égale au rayon spectrale), enfin qu'elle dépend de façon analytique de $\mu$. Le point clef pour démontrer cette dernière propriété est le fait que c'est une valeur propre simple. 
Il reste alors à identifier cette valeur propre dans un espace $L^{2}$ avec poids à une valeur propre du vrai opérateur $H_{\mu}^{-1}$ agissant sur $E_{0}$ (pour $\mu$ positif bien sûr). On utilise un résultat ad hoc basé sur le simple fait qu'il $y$ a dans $E_{0}$ des fonctions positives sur le demi-axe imaginaire pur négatif (Proposition 2, Sect. 5 du présent article).

\section{Précisions sur l'espace de Bargmann}

Notation 3. Nous poserons:

$$
\varphi_{k}(z)=\frac{(i z)^{k}}{\sqrt{k !}} .
$$

Les $\varphi_{k}$ forment une base orthonormée de l'espace de Bargmann.

Lemme 1 (Intissar [3]). Si $f \in E$, l'intégrale:

$$
\int_{\mathbb{C}} \frac{e^{-|z|^{2}}}{1+|z|^{2}}\left|f^{\prime}(z)\right|^{2} d x d y
$$

est convergente.

Lemme 2. Soit $f$ analytique complexe et de carré intégrable sur la bande

$$
\mathscr{B}=\{z ; \mathscr{R} z \in] a, b[\} .
$$

Alors pour tout $x \in] a, b[$, l'intégrale:

est convergente.

$$
\int_{-\infty}^{+\infty}|f(x+i y)|^{2} d y
$$

Démonstration. Si on remplace "tout $x$ " par "presque tout $x$ ", le lemme est une simple application du théorème de Fubini.

Soit $x \in] a, b\left[\right.$, choisissons $a^{\prime}, b^{\prime}$ tels que:

$$
a<a^{\prime}<x<b^{\prime}<b,
$$

les intégrales $\int_{-\infty}^{+\infty}\left|f\left(a^{\prime}+i y\right)\right|^{2} d y$ et $\int_{-\infty}^{+\infty}\left|f\left(b^{\prime}+i y\right)\right|^{2} d y$ convergent.

On a pour tout $T$ assez grand:

$$
\begin{aligned}
2 i \pi f(x+i y)= & \int_{-T}^{T} \frac{f\left(b^{\prime}+i v\right)}{b^{\prime}-x+i(v-y)} d v-\int_{a^{\prime}}^{b^{\prime}} \frac{f(u+i T)}{u-x+i(T-y)} d u \\
& -\int_{-T}^{T} \frac{f\left(a^{\prime}+i v\right)}{a^{\prime}-x+i(v-y)} d v+\int_{a^{\prime}}^{b^{\prime}} \frac{f(u-i T)}{u-x-i(T+y)} d u,
\end{aligned}
$$

puis en prenant $h$ assez petit:

$$
\begin{aligned}
2 i \pi f(x+i y)= & \frac{1}{h} \int_{-h / 2}^{h / 2} d s \int_{-s^{-}-T}^{s+T}\left[\frac{f\left(b^{\prime}+i v\right)}{b^{\prime}-x+i(v-y)}-\frac{f\left(a^{\prime}+i v\right)}{a^{\prime}-x+i(v-y)}\right] d v \\
& +\frac{1}{h} \int_{-h / 2}^{h / 2} d s \int_{a^{\prime}}^{b^{\prime}}\left[\frac{f(u-i s-i T)}{u-x-i(s+y+T)}-\frac{f(u+i s+i T)}{u-x+i(T+s-y)}\right] d u .
\end{aligned}
$$


Quand $T$ tend vers l'infini, l'intégrale interne de la première ligne tend vers l'intégrale sur toute la droite en restant bornée; la deuxième ligne tend vers zéro puisque la fonction:

$$
(u, s) \mapsto \frac{f(u+i s)}{u-x+i(s-y)}
$$

est intégrable sur $] a^{\prime}, b^{\prime}[\times \mathbb{R}$. D'où

$$
f(x+i y)=\frac{1}{2 i \pi} \int_{-\infty}^{+\infty}\left[\frac{f\left(b^{\prime}+i v\right)}{b^{\prime}-x+i(v-y)}-\frac{f\left(a^{\prime}+i v\right)}{a^{\prime}-x+i(v-y)}\right] d v .
$$

La convolution d'une fonction de carré intégrable avec $1 /(c+i y)$ donne encore une fonction de carré intégrable, remarque qui achève la démonstration.

Lemme 3. Si $f \in E$, les intégrales

$$
\int_{-\infty}^{+\infty}|f(x+i y)|^{2} e^{-y^{2}} d y \quad \text { et } \int_{-\infty}^{+\infty}\left|f^{\prime}(x+i y)\right|^{2} \frac{e^{-y^{2}}}{1+y^{2}} d y
$$

sont convergentes pour tout $x$.

Démonstration. On applique le lemme 2 à $f(z) e^{z^{2} / 2}$ et $f^{\prime}(z) e^{z^{2} / 2} /(a+z)$, où a est un nombre réel convenablement choisi.

Remarque. Si on pose $P f(y)=f(-i y)$, il résulte de ce lemme que $P$ est une application, injective d'après le principe de prolongement analytique, de $E_{0}$ dans $L_{0}^{2}=L^{2}(] 0, \infty\left[, e^{-x^{2}} d x\right)$.

L'injection ainsi définie est continue. Pour le montrer, il suffit de vérifier que son graphe est fermé. Soit donc $\left(u_{n}\right)$ une suite de $E_{0}$ telle que

$$
\begin{aligned}
\lim u_{n} & =w\left(\operatorname{dans} E_{0}\right), \\
\lim P u_{n} & =v\left(\operatorname{dans} L_{0}^{2}\right) .
\end{aligned}
$$

Toute fonction $\varphi$ mesurable à support borné sur $] 0, \infty[$ définit une forme continue sur $E_{0}$ par la formule:

$$
\langle\varphi, u\rangle=\int_{0}^{\infty} \varphi(y) u(-i y) d y,
$$

on a donc

$$
\lim \left\langle\varphi, u_{n}\right\rangle=\int_{0}^{\infty} \varphi(y) P w(y) d y
$$

et on a aussi:

$$
\lim \int_{0}^{\infty} \varphi(y) P u_{n}(y) d y=\int_{0}^{\infty} \varphi(y) v(y) d y .
$$

Mais

$$
\left\langle\varphi, u_{n}\right\rangle=\int_{0}^{\infty} \varphi(y) P u_{n}(y) d y,
$$


d'où:

$$
P w=v . \quad \text { c.q.f.d. }
$$

\section{Restriction au demi-axe imaginaire et inversion de $\boldsymbol{H}_{\mu}$}

Nous supposons $\mu>0$ dans cette partie 3 .

Son but est d'expliciter l'inverse de $H_{\mu}$, du moins pour les valeurs imaginaires pures "négatives" sous la forme:

$$
H_{\mu}^{-1} f(-i y)=\int_{0}^{\infty} N_{\mu}\left(y, y^{\prime}\right) f\left(-i y^{\prime}\right) d y^{\prime} .
$$

En notant $H_{\mu}^{-1} f=u$, on a

$$
H_{\mu} u(z)=i z u^{\prime \prime}(z)+z(i z+\mu) u^{\prime}(z)=f(z),
$$

et si on pose

$$
\tilde{u}(y)=u(-i y), \tilde{f}(y)=f(-i y),
$$

il vient:

$$
-y \tilde{u}^{\prime \prime}(y)+y(y+\mu) \tilde{u}^{\prime}(y)=\tilde{f}(y) .
$$

On peut tirer de là:

$$
\tilde{u}^{\prime}(y)=\left[c+\int_{y}^{\infty} e^{-\frac{1}{2} z^{2}-\mu z} \tilde{f}(z) \frac{d z}{z}\right] e^{\frac{1}{2} y^{2}+\mu y},
$$

où l'intégrale converge d'après le lemme 3.

Montrons que la constante $c$ est nulle. Toujours d'après le lemme 3, nous savons que $\tilde{u}^{\prime}$ est de carré intégrable pour la mesure $\frac{e^{-y^{2}}}{1+y^{2}} d y$. Or $e^{\frac{1}{2} y^{2}+\mu y}$ ne l'est pas. Il faudra donc que $c$ soit nulle si:

$$
e^{\mu y} \int_{y}^{\infty} e^{-\frac{1}{2} z^{2}-\mu z} \tilde{f}(z) \frac{d z}{z}
$$

est de carré intégrable. Or on a d'après Cauchy-Schwartz:

$$
\begin{aligned}
\left|\int_{y}^{\infty} e^{-\frac{1}{2} z^{2}-\mu z} \tilde{f}(z) \frac{d z}{z}\right|^{2} & \leqq \int_{0}^{\infty}|\tilde{f}(z)|^{2} e^{-z^{2}} d z \int_{y}^{\infty} e^{-2 \mu z} \frac{d z}{z^{2}} \\
& \leqq \int_{0}^{\infty}|\tilde{f}(z)|^{2} e^{-z^{2}} d z \frac{e^{-2 \mu y}}{2 \mu y^{2}},
\end{aligned}
$$

ce qui assure la convergence à l'infini, et aussi

$$
\left|\int_{y}^{\infty} e^{-\frac{1}{2} z^{2}-\mu z} \tilde{f}(z) \frac{d z}{z}\right|^{2} \leqq \int_{0}^{\infty}|\tilde{f}(z)|^{2} \frac{e^{-z^{2}}}{z^{2}} d z \frac{e^{-2 \mu y}}{2 \mu},
$$

où l'intégrale du second membre converge puisque $\tilde{f}$ est analytique et nulle à l'origine. 
Maintenant nous connaissons $\tilde{u}^{\prime}$ et nous savons que $\tilde{u}(0)=0$ d'où:

$$
\tilde{u}(y)=\int_{0}^{y} e^{\frac{1}{2} y_{1}^{2}+\mu y_{1}} d y_{1} \int_{y_{1}}^{\infty} \frac{e^{-\frac{1}{2} y_{2}^{2}-\mu y_{2}}}{y_{2}} \tilde{f}\left(y_{2}\right) d y_{2} .
$$

Si on complète ceci par une application du théorème de Fubini, on peut résumer le résultat de ces calculs de la façon suivante:

Lemme 4. On a, pour $\mu>0, v \in E_{0}$ et $y>0$ :

$$
\begin{aligned}
H_{\mu}^{-1} v(-i y) & =\int_{0}^{\infty} \Phi_{\mu}\left(\min \left(y, y^{\prime}\right)\right) \frac{e^{-\frac{1}{2} y^{\prime 2}-\mu y^{\prime}}}{y^{\prime}} v\left(-i y^{\prime}\right) d y^{\prime} \\
& =\int_{0}^{y} e^{\frac{1}{2} y_{1}^{2}+\mu y_{1}} d y_{1} \int_{y_{1}}^{\infty} \frac{e^{-\frac{1}{2} y_{2}^{2}-\mu y_{2}}}{y_{2}} v\left(-i y_{2}\right) d y_{2},
\end{aligned}
$$

où on a posé:

$$
\Phi_{\mu}(y)=\int_{0}^{y} e^{\frac{1}{2} y_{1}^{2}+\mu y_{1}} d y_{1}
$$

Nous confondrons désormais $E_{0}$ avec l'espace des fonctions définies sur $\mathbb{R}^{+}$ par $u(-i y)$ où $y \in E_{0}$, sans changer sa norme.

Pour tout nombre complexe $\mu$ nous noterons $K_{u}$ l'opérateur intégral de noyau:

$$
\begin{aligned}
N_{\mu}\left(y, y^{\prime}\right) & =\Phi_{\mu}\left(\min \left(y, y^{\prime}\right)\right) \frac{e^{-\frac{1}{2} y^{\prime 2}-\mu y^{\prime}}}{y^{\prime}} \\
& =\frac{e^{-\frac{1}{2} y^{\prime 2}}}{y^{\prime}} \int_{0}^{y} \theta\left(y^{\prime}-y_{1}\right) e^{\frac{1}{2} y_{1}^{2}} e^{-\mu\left(y^{\prime}-y_{1}\right)} d y_{1},
\end{aligned}
$$

où $\theta$ désigne une fonction nulle pour $y$ négatif et valant 1 pour $y$ positif.

Nous avons vu que cet opérateur agissait sur $E_{0}$ (au sens des restrictions comme il est dit ci-dessus) pour $\mu>0$ mais nous allons voir qu'il agit aussi ( $y$ compris pour d'autres valeurs de $\mu$ ) sur une famille d'espaces $L^{2}$ avec poids:

Dégageons pour la référence ultérieure deux résultats évidents sur la deuxième forme de $N_{\mu}$.

Lemme 5. Pour $\mu$ réel, $N_{\mu}$ est une fonction décroissante de $\mu$. Si $\mu=\mu^{\prime}+i \mu^{\prime \prime}\left(\mu^{\prime}\right.$ et $\mu^{\prime \prime}$ réels) on $a$ :

$$
\left|N_{\mu}\left(y, y^{\prime}\right)\right| \leqq N_{\mu^{\prime}}\left(y, y^{\prime}\right) .
$$

Nous aurons aussi besoin du comportement asymptotique de $\Phi_{\mu}$ :

Lemme 6. Supposons $\mu$ réel

(a) $\Phi_{\mu}(y) \sim y(y \rightarrow 0)$,

(b) Pour $y>y_{0}>-\mu$, on $a$ :

$$
\Phi_{\mu}(y) \leqq \Phi_{\mu}\left(y_{0}\right)+\frac{4 e^{\frac{1}{2} y^{2}+\mu y}}{y+y_{0}+2 \mu} .
$$


Démonstration. (a) est une simple constatation.

$$
\begin{aligned}
\Phi_{\mu}(y)-\Phi_{\mu}\left(y_{0}\right) & \leqq 2 \int_{\left(y+y_{0}\right) / 2}^{y} e^{\frac{1}{2} y_{1}^{2}+\mu y_{1}} \frac{\left(y_{1}+\mu\right)}{y_{1}+\mu} d y_{1} \\
& \leqq \frac{4}{y+y_{0}+2 \mu} e^{\frac{1}{2} y^{2}+\mu y} .
\end{aligned}
$$

\section{Extension de $K_{\mu}$ à un espace $L^{2}$ avec poids}

Pour tout réel $\alpha$ nous poserons

$$
L_{\alpha}^{2}=L^{2}\left(\mathbb{R}^{+}, e^{-x^{2}-2 \alpha x} d x\right)
$$

(espace des fonctions de carré intégrable par rapport à la mesure $\left.\exp \left(-x^{2}-2 \alpha x\right) d x\right)$.

Lemme 7. Pour tout $\mu$ de partie réelle au moins égale à $\alpha, K_{\mu}$ s'étend en un opérateur de Hilbert-Schmidt de $L_{\alpha}^{2}$ dans lui-même.

Démonstration. $K_{\mu}$ s'écrit:

$$
K_{\mu} u(y)=\int_{0}^{\infty} \tilde{N}_{\mu}\left(y, y^{\prime}\right) u\left(y^{\prime}\right) e^{-y^{\prime 2}-2 \alpha y^{\prime}} d y^{\prime},
$$

où

$$
\tilde{N}_{\mu}\left(y, y^{\prime}\right)=N_{\mu}\left(y, y^{\prime}\right) e^{y^{\prime 2}+2 \alpha y^{\prime}} .
$$

Il sera de Hilbert-Schmidt dans $L_{\alpha}^{2}$ si la fonction $\tilde{N}_{\mu}$ est de carré intégrable par rapport à la mesure:

$$
e^{-y^{2}-2 \alpha y} e^{-y^{\prime 2}-2 \alpha y^{\prime}} d y d y^{\prime} .
$$

D'après le lemme $5,\left|\widetilde{N}_{\mu}\right| \leqq \tilde{N}_{\alpha}$ de sorte qu'il suffit de faire la démonstration pour $\mu=\alpha$.

On est alors ramené à la convergence de l'intégrale

$$
I=\int_{0}^{\infty} \int_{0}^{\infty} \Psi\left(y, y^{\prime}\right) d y d y^{\prime}
$$

où

$$
\begin{aligned}
\Psi\left(y, y^{\prime}\right) & =\tilde{N}_{\alpha}^{2} e^{-y^{2}-2 \alpha y} e^{-y^{\prime 2}-2 \alpha y^{\prime}} \\
& =\Phi_{\alpha}^{2}\left(\min y, y^{\prime}\right) \frac{e^{-y^{2}-2 \alpha y}}{y^{\prime 2}} .
\end{aligned}
$$

Une application du théorème de Fubini donne: $I=I_{-}+I_{+}$

$$
I_{-}=\int_{0}^{\infty} \frac{d y^{\prime}}{y^{\prime 2}} \int_{0}^{y^{\prime}} \Phi_{\alpha}^{2}(y) e^{-y^{2}-2 \alpha y} d y .
$$


D'après le lemme 6 l'intégrale en $d y$ se comporte en $y^{\prime 3}$ quand $y^{\prime}$ tend vers 0 et a une limite quand $y^{\prime}$ tend vers l'infini, d'où la convergence de $I_{-}$

$$
I_{+}=\int_{0}^{\infty} \frac{d y^{\prime}}{y^{\prime 2}} \Phi_{\alpha}^{2}\left(y^{\prime}\right) \int_{y^{\prime}}^{\infty} e^{-y^{2}-2 \alpha y} d y .
$$

D'après le lemme 6 , partie (a), la fonction à intégrer par rapport à $y^{\prime}$ est continue au point $y^{\prime}=0$. D'autre part, pour $y^{\prime}$ assez grand:

$$
\int_{y^{\prime}}^{\infty} e^{-y^{2}-2 \alpha y} d y \leqq \frac{e^{-y^{\prime 2}-2 y^{\prime} \alpha}}{2\left(y^{\prime}+\alpha\right)} .
$$

Compte tenu du lemme 6 partie (b), la fonction à intégrer est donc en $y^{\prime-5}$ pour $y^{\prime}$ tendant vers l'infini; Il en résulte que $I_{+}$est elle aussi convergente ce qui achève la démonstration du lemme.

Remarque. Cette démonstration permet d'apporter une précision supplémentaire; la norme de Hilbert-Schmidt de $K_{\mu}$ (comme opérateur sur $L_{\alpha}^{2}$ ) est majorée indépendamment de $\mu$ pour $\mathscr{R} \mu>\alpha$.

Lemme 8. Lapplication $\mu \mapsto K_{\mu}$ est analytique sur le demi-plan $\mathscr{R} \mu>\alpha$ au sens de la norme de Hilbert-Schmidt des opérateurs sur $L_{\alpha}^{2}$ (et a fortiori au sens de $\mathscr{L}\left(L_{\alpha}^{2}\right)$ ).

Démonstration. Compte tenu de la remarque qui précède, il suffit de démontrer l'analyticité de l'application:

$$
\mu \mapsto\left(u, K_{\mu} v\right)_{L_{\alpha}^{2}}
$$

pour $u$ et $v$ appartenant à $L_{\alpha}^{2}$ (Kato [7, VII. 1.1, p. 365], le résultat remonte autant que je sache à Grothendieck [2]).

Démontrons d'abord la continuité. On a:

$$
\left(u, K_{\mu} v\right)_{L_{\alpha}^{2}}=\int_{0}^{\infty} e^{-x^{2}-2 \alpha x} \overline{u(x)} d x \int_{0}^{\infty} N_{\mu}(x, y) v(y) d y .
$$

La fonction à intégrer en $d x d y$ est majorée en module par:

$$
e^{-x^{2}-2 \alpha x} N_{\alpha}(x, y)|u(x)||v(y)|,
$$

la continuité résulte donc du théorème de convergence majorée.

Pour démontrer l'analyticité, il suffit maintenant de prouver que pour tout circuit fermé $\gamma$ du demi-plan, on a:

$$
\int_{\gamma}\left(u, K_{\mu} v\right)_{L_{\alpha}^{2}} d \mu=0 .
$$

La majoration déjà utilisée permet d'appliquer le théorème de Fubini, ce qui met l'intégrale sous la forme:

$$
\int_{0}^{\infty} \int_{0}^{\infty} d x d y e^{-x^{2}-2 \alpha x} \bar{u}(x) v(y) \int_{\gamma} K_{\mu}(x, y) d \mu,
$$

où l'intégrale interne est nulle d'après l'analyticité en $\mu$ de $K_{\mu}$. 


\section{Rappels sur les valeurs propres simples des opérateurs compacts}

On sait que le spectre d'un opérateur compact est formé, outre 0, de valeurs propres isolées de multiplicité finie. Disons que la valeur propre $\sigma \neq 0$ de l'opérateur $T$ est simple si cette multiplicité est un, c'est-à-dire si les sous-espaces vectoriels $(T-\sigma)^{-k}(0)$ sont tous de dimension un (et par conséquent identiques entre eux).

Il est équivalent de dire que le projecteur spectral $P_{\sigma}$ associé à $\sigma$ est de rang un. La restriction de $T-\sigma$ à l'hyperplan $P_{\sigma}^{-1}(0)$ est inversible. On a:

$$
P_{\sigma}(x)=\left\langle u^{*}, x\right\rangle u,
$$

où $u$ et $u^{*}$ sont des vecteurs propres de $T$ et $T^{*}$ respectivement associés aux valeurs propres $\sigma$ et $\bar{\sigma}$ (Kato [7, III.6.7, Théorème 6.26 et III.6.5, p. 181]).

Si la multiplicité géométrique de $\sigma$ est un, c'est-à-dire s'il n'y a qu'un vecteur propre à un coefficient scalaire près, et si de plus la résolvante $(T-z)^{-1}$ présente en $\sigma$ un pôle simple, alors $\sigma$ est une valeur propre simple (Yosida [11, VIII.8, Théorème 3]).

Nous utiliserons encore la version suivante (simplifiée) du théorème de KatoRellich:

Soient $E$ un espace de Banach sur $\mathbb{C}, F$ une fonction analytique d'une variable complexe à valeurs dans $\mathscr{L}(E)$ muni de sa norme et $\sigma_{0}$ une valeur propre simple de $F\left(\mu_{0}\right)$. Il existe des voisinages $U$ et $V$ de $\mu_{0}$ et $\sigma_{0}$ tels que pour tout $\mu \in U, F(\mu)$ possède une valeur propre unique $\sigma(\mu)$ appartenant à $V$ et la fonction $\sigma$ est analytique (de plus $\sigma(\mu)$ est une valeur propre simple). (Reed et Simon [9, Théorème XII.8, compte tenu de la remarque qui suit la définition d'une famille analytique p. 14; noter que nos valeurs propres simples sont ce que ces auteurs appellent des "non-degenerate eigenvalues" définies 1.c. p. 13]).

Nous utiliserons une version généralisée et précisée du théorème de Jentzsch [6]:

Soit $T$ un opérateur compact sur $L^{p}(\mu)$ où $\mu$ est une mesure $\sigma$-finie. Supposons que $T$ soit donné par un noyau $\mu \otimes \mu$ presque partout strictement positif. Alors $T$ possède au moins une valeur propre non nulle. Une seule de ses valeurs propres a pour module le rayon spectral de $T$. Cette valeur propre est positive, simple et il lui correspond un vecteur propre presque partout strictement positif.

C'est le théorème V.6.6 de Schäfer [10, p. 337] à quelques détails près. La version donnée ici est simplifiée par particularisation. D'autre part Schäfer n'énonce pas que le rayon spectral est une valeur propre simple, mais seulement qu'il lui correspond un vecteur propre unique à un coefficient près. Cependant sa démonstration prouve aussi que c'est un pôle simple de la résolvante.

Corollaire 1. Soit $\alpha$ un nombre réel. Pour $\mu \geqq \alpha, K_{\mu}$ considéré comme opérateur sur $L_{\alpha}^{2}$ possède au moins une valeur propre non nulle. La plus grande en module de ses valeurs propres est simple, positive, il lui correspond un vecteur propre presque partout strictement positif sur $\mathbb{R}^{+}$.

Corollaire 2. Sous les hypothèses du théorème de Jentzsch et avec les mêmes notations, soit $v$ un vecteur positif non nul dans $L^{p}(\mu)$. Notons $r$ le rayon spectral de $T$ 
et $P_{r}$ le projecteur spectral associé à la valeur propre $r$. On a:

$$
\lim _{n \rightarrow \infty} r^{-n} T^{n} v=P_{r} v \neq 0 .
$$

Démonstration. Montrons d'abord que $P_{r} v \neq 0$. On a

$$
P_{r} v(x)=u(x) \int u^{*}(y) v(y) d \mu(y)
$$

où $u$ et $u^{*}$ sont des vecteurs propres de $T$ et $T^{*}$ respectivement associés tous deux à la valeur propre $r$.

Les spectres de $T$ et $T^{*}$ étant complexes conjugués, $r$ est le rayon spectral de $T^{*}$. Ce dernier a pour noyau le transposé du noyau de $T$, qui est presque partout strictement positif. On peut donc lui appliquer aussi le théorème de Jentzsch. Il en résulte que $u^{*}$ est presque partout strictement positif d'où enfin:

$$
\int u^{*}(y) v(y) d y>0
$$

qui entraîne la non nullité de $P_{r} v$.

Notons $T^{\prime}$ la restriction de $T$ à $P_{r}^{-1}(0)$ et $r^{\prime}$ son rayon spectral. $r$ n'est pas une valeur propre de $T^{\prime}$ et comme toutes les autres valeurs propres de $T$ sont de module strictement plus petit il vient $r^{\prime}<r$, d'où

$$
\lim _{n \rightarrow \infty} r^{-n} T^{\prime n} w=0
$$

pour tout $w$ tel que $P_{r} w=0$.

Or on a

$$
r^{-n} T^{n} v=P_{r} v+r^{-n} T^{\prime n}\left(v-P_{r} v\right) .
$$

Proposition 2. Sous les hypothèses du théorème de Jentzsch et avec les mêmes notations, soit $E$ un espace de Banach plongé dans $L^{p}(\mu)$ avec une norme plus forte. Supposons

a) que T laisse $E$ invariant et soit compact en tant qu'opérateur sur $E$,

b) qu'il existe dans $E$ une fonction positive non nulle.

Alors le rayon spectral de T en tant qu'opérateur sur E est le même que son rayon spectral en tant qu'opérateur sur $L^{p}(\mu)$.

Démonstration. Le spectre de $T$ comme opérateur sur $E$ étant formé de 0 et de valeurs propres est contenu dans son spectre comme opérateur sur $L^{p}(\mu)$. Notant $r^{\prime}$ le rayon spectral de l'opérateur sur $E$ et $r$ celui de l'opérateur sur $L^{p}(\mu)$, on a donc: $r^{\prime} \leqq r$. Montrons que l'inégalité ne peut pas être stricte. On aurait alors pour toute $v$ $\in E$ :

$$
\lim _{n \rightarrow \infty} r^{-n} T^{n} v=0
$$

au sens de $E$ et a fortiori au sens de $L^{p}(\mu)$. Mais si $v$ est positive celà contredit le corollaire $2 \mathrm{du}$ théorème de Jentzsch.

Corollaire. Sous les hypothèses de la Proposition 2, appelons $r$ le rayon spectral de $T$. $C$ 'est une valeur propre simple de $T$ comme opérateur sur $E$, la seule de module maximal, il lui correspond les mêmes vecteurs propres dans $E$ que dans $L^{p}(\mu)$. 
(Ce corollaire ne sera pas utilisé dans ce qui suit.)

Nous aurons besoin encore d'un résultat connu, à savoir que le rayon spectral d'un opérateur à noyau positif est fonction croissante dudit noyau.

Proposition 3. Soient $T_{1}$ et $T_{2}$ deux opérateurs intégraux positifs sur $L^{p}(\mu)$. Si le noyau de $T_{1}$ est plus petit que celui de $T_{2}$ alors le rayon spectral de $T_{1}$ est plus petit ou égal à celui de $T_{2}$.

Démonstration. Si $S$ est un opérateur intégral, notons $\tilde{S}$ son noyau.

Remarquons d'abord que si $\tilde{S}$ est positif on a:

$$
|S u| \leqq S|u|
$$

d'où on déduit:

d'abord que si $\tilde{S}$ est positif alors

$$
\|S\|=\sup \{\|S u\| ; u \geqq 0 \quad \text { et } \quad\|u\|=1\},
$$

et ensuite que si $0 \leqq \tilde{S} \leqq \tilde{T}$ alors $\|S\| \leqq\|T\|$.

D'après la formule qui donne le noyau du produit de deux opérateurs intégraux on a pour tout $n \widetilde{T}_{1}^{n} \leqq \widetilde{T}_{2}^{n}$, d'où $\left\|T_{1}^{n}\right\| \leqq\left\|T_{2}^{n}\right\|$, et comme le rayon spectral de $T_{j}$ est la limite de $\left(\left\|T_{j}^{n}\right\|\right)^{1 / n}$ notre proposition en résulte.

\section{Démonstration de la proposition 1}

Soit $\alpha$ un nombre réel quelconque. Pour $\mu \geqq \alpha$, désignons par $\sigma_{\alpha}(\mu)$ le rayon spectral de $K_{\mu}$ considéré comme opérateur sur $L_{\alpha}^{2}$. C'est un nombre strictement positif, valeur propre simple de $K_{\mu}$ considéré comme opérateur sur $L_{\alpha}^{2}$ (corollaire $1 \mathrm{du}$ théorème de Jentzsch). De plus $K_{\mu}$ dépend analytiquement de $\mu$ (lemme 8), on peut donc appliquer le théorème de Kato-Rellich: $\sigma_{\alpha}$ est une fonction analytique réelle sur $] \alpha,+\infty$ [. D'après le lemme 5 , le noyau de $K_{\mu}$ est fonction décroissante de $\mu$ donc (proposition 3) $\sigma_{\alpha}$ est une fonction décroissante.

D'après la proposition 2 on a $\sigma_{\alpha}(\mu)=\sigma_{\beta}(\mu)$ pour $\mu \geqq \max (\alpha, \beta)$. Les $\sigma_{\alpha}$ définissent ainsi une même fonction $\sigma$ strictement positive, analytique réelle et décroissante sur $\mathbb{R}$ tout entier.

Prenons maintenant $\mu$ positif.

$E_{0}$ est continûment plongé dans $L_{0}^{2}$ (lemme 3 et la remarque qui le suit). $K_{\mu}$ est un opérateur compact sur $E_{0}$, c'est en tant que tel que nous l'avons construit (propriété $c$ de $H_{\lambda, \mu}$ et lemme 4). Enfin $E_{0}$ possède des fonctions qui sont positives sur le demi-axe imaginaire négatif (et non nulles) par exemple $\frac{\sin ^{2} i z}{i z}$. Toutes les hypothèses de la proposition 2 sont donc vérifiées et par conséquent $\sigma(\mu)$ est aussi le rayon spectral de $K_{\mu}$ considéré comme opérateur sur $E_{0}$. Cet opérateur étant compact à valeurs propres positives (propriétés $e$ et $f$ de $H_{\lambda, \mu}$ ) c'est aussi sa plus grande valeur propre.

En posant $E(\mu)=1 / \sigma(\mu)$, et en se souvenant que les valeurs propres de $H_{\mu}$ sont les inverses de celles de $K_{\mu}$ considéré comme opérateur sur $E_{0}$, on vérifie toutes les propriétés annoncées dans la proposition 1. 


\section{Quelques remarques sur le cas limite $\boldsymbol{\mu}=0$}

$H_{0}=i A^{*}\left(A+A^{*}\right) A$ est formellement anti-adjoint. On s'attendait donc au départ à ce que $E(0)$ soit nul. On a vu qu'il était strictement positif. D'autre part pour $\mu$ strictement négatif, $E(\mu)$ n'est certes plus une valeur propre de $H_{\mu}$ : la plus grande valeur propre de ce dernier est $-E(-\mu)$.

Rappelons les notations introduites au Sect. 1: $H_{0}$ a comme domaine

$$
D=\left\{f ; f \in E_{0}, H_{0} f \in E_{0}\right\} .
$$

Si on limite le domaine aux polynômes et qu'on prend la fermeture de l'opérateur obtenu on la note $H_{0}^{\min }$ dont $H_{0}$ est évidemment une extension. Contrairement à ce qui se passe pour $\mu \neq 0$, nous allons voir que ces deux opérateurs sont différents.

Lemme 9. $K_{0}$ est un opérateur compact dans $E_{0}$.

Démonstration. La technique est d'utiliser le développement dans la base des $\varphi_{k}$ (à des coefficients près, c'est le développement de Taylor).

Posons:

$$
K_{0} \varphi_{k}=\psi_{k},
$$

et démontrons d'abord que $\psi_{k} \in E_{0}$, à commencer par $\psi_{1}$.

$$
\begin{aligned}
\psi_{1}(y) & =\int_{0}^{y} e^{\frac{1}{2} y_{1}^{2}} d y_{1} \int_{y_{1}}^{\infty} e^{-\frac{1}{2} y_{2}^{2}} d y_{2} \\
& =-\int_{0}^{y} d y_{1} \int_{0}^{y_{1}} e^{-\frac{1}{2}\left(y_{2}^{2}-y_{1}^{2}\right)} d y_{2}+\omega,
\end{aligned}
$$

où on a posé:

$$
\begin{aligned}
& \omega=\sqrt{2 \pi} \int_{0}^{y} e^{-\frac{1}{2} y_{1}^{2}} d y_{1}=\sum_{k=0}^{\infty}(-1)^{k} a_{k} \varphi_{2 k+1}, \\
& a_{k}=\frac{\sqrt{(2 k) ! \pi / 2}}{2^{k} k ! \sqrt{2 k+1}} \sim \frac{1}{2} k^{-3 / 4} \pi^{1 / 4} .
\end{aligned}
$$

La dernière relation montre que $\omega$ est bien dans l'espace de Bargmann. Passons au premier terme:

$$
\begin{aligned}
\int_{0}^{y_{1}} e^{-\frac{1}{2}\left(y_{2}^{2}-y_{1}^{2}\right)} d y_{2} & =\sum_{k=0}^{\infty} \frac{y_{1}^{2 k+1}}{2^{k} k !} \int_{0}^{1}\left(1-t^{2}\right)^{k} d t \\
& =\sum_{k=0}^{\infty} \frac{2^{k} k !}{\sqrt{(2 k+1) !}} \varphi_{2 k+1}\left(y_{1}\right),
\end{aligned}
$$

d'où

$$
\begin{aligned}
\int_{0}^{y} d y_{1} \int_{0}^{y_{1}} e^{-\frac{1}{2}\left(y_{2}^{2}-y_{1}^{2}\right)} d y_{2} & =\sum_{k=0}^{\infty} b_{k} \varphi_{2(k+1)}(y) \\
b_{k} & =\frac{2^{k} k !}{\sqrt{2(k+1)(2 k+1) !}} \sim \frac{\pi^{1 / 4} k^{-3 / 4}}{2} .
\end{aligned}
$$


C'est encore une suite de carré sommable.

Une intégration par parties fournit la relation de récurrence:

$$
\psi_{k+1}=\frac{\varphi_{k}}{k \sqrt{k+1}}+\frac{k-1}{\sqrt{k(k+1)}} \psi_{k-1} .
$$

D'où l'on tire:

où

$$
\psi_{k}=P_{k-1}+\alpha_{k} \psi_{1}
$$

$$
\begin{aligned}
\alpha_{2 k} & =0, \\
\alpha_{2 k+1} & =\sqrt{2} b_{k}, \\
P_{0} & =0 \\
P_{1} & =\varphi_{1} / \sqrt{2}, \\
P_{k} & =\frac{k-1}{\sqrt{k(k+1)}} P_{k-2}+\frac{\varphi_{k}}{k \sqrt{k+1}} .
\end{aligned}
$$

En posant $\left\|P_{k}\right\|^{2}=p_{k}$ et en tenant compte de l'orthogonalité de $P_{k-2}$ et de $\varphi_{k}$, on obtient:

$$
p_{k}=\frac{(k-1)^{2}}{k(k+1)} p_{k-2}+\frac{1}{k^{2}(k+1)},
$$

d'où on peut tirer:

$$
p_{k}=0\left(k^{-3 / 2}\right) .
$$

Nous pouvons maintenant montrer que $K_{0}$ est limite en norme d'opérateurs de rang fini.

Soit

$$
f=\sum_{k=1}^{\infty} c_{k} \varphi_{k} .
$$

On a, au moins au sens de la convergence compact:

$$
K_{0} f=\sqrt{2}\left(f, \omega-\psi_{1}\right) \psi_{1}+\sum_{k=1}^{\infty} c_{k} P_{k-1} .
$$

Majorons par Cauchy-Schwarz le reste de la série

$$
\left\|\sum_{k=n+1}^{\infty} c_{k} P_{k-1}\right\| \leqq \sum_{k=n+1}^{\infty}\left|c_{k}\right|\left\|P_{k-1}\right\| \leqq\|f\|\left(\sum_{k=n}^{\infty} p_{k}\right)^{1 / 2} .
$$

Quelques propriétés de $H_{0}$ et $H_{0}^{\mathrm{min}}$ :

a) $H_{0}^{\min }$ est antisymétrique,

b) $K_{0}$ est un inverse à droite de $H_{0}$ (on le vérifie par un calcul direct).

Conséquences. $H_{0}$ est surjectif et $E(0)$ est une valeur propre de $H_{0}$ (on montre comme dans la démonstration de la proposition 1 que $\sigma(0)$ est une valeur propre de $K_{0}$ considéré comme opérateur sur $E_{0}$ ) 
c) $H_{0}$ n'est pas anti-symétrique.

Il y a à celà deux raisons dont chacune suffirait. D'abord il a une valeur propre réelle non nulle. Ensuite il est surjectif (puisqu'il a un inverse à droite) mais non injectif (puisqu'il annule la fonction $\omega$ qui intervient dans la démonstration du lemme 9).

Pour avoir dans un certain sens un opérateur limite des $H_{\mu}$ pour $\mu$ positif tendant vers 0 on essaiera de prendre comme domaine l'image de $K_{0}$, notons la $D_{0}$. Celà est possible car $D_{0}$ est dense. Mais on n'obtient pas une extension de $H_{0}^{\text {min }}$, en d'autres termes $D_{0}$ ne contient pas tous les polynômes nuls à l'origine.

Montrons que $D_{0}$ est dense. Pour celà, prenons une fonction $u \in E_{0}$ orthogonale à $D_{0}$. Elle est en particulier orthogonale à tous les $\psi_{k}$ (notations de la démonstration du lemme 9), à commencer par $\psi_{1}$. On a d'après la formule (a) de la démonstration du lemme 9:

$$
\left(P_{k}, u\right)=\left(\psi_{k+1}, u\right)=0 .
$$

Comme $P_{k}$ est de degré $k$ exactement l'ensemble des $P_{k}$ est une base algébrique de l'espace vectoriel des polynômes nuls à l'origine, il est donc total dans $E_{0}$ d'où $u=0$.

Montrons maintenant que tous les polynômes nuls à l'origine ne sont pas dans $D_{0}$. Soit $P \in D_{0}$ un polynôme. On a par définition:

$$
P=K_{0} Q, Q \in E_{0} \text {, }
$$

donc

$$
H_{0} P=H_{0} K_{0} Q=Q,
$$

ce qui montre que $Q$ est lui-même un polynôme. Posons:

$$
Q=\sum_{k=1}^{n} a_{k} \varphi_{k}
$$

Il vient (toujours la formule (a))

$$
P=\sum_{k=1}^{n} a_{k} P_{k-1}+\sum_{k=1}^{n} a_{k} \alpha_{k} \psi_{1} .
$$

Comme $\psi_{1}$ n'est pas un polynôme on en déduit

$$
\sum_{k=1}^{n} a_{k} \alpha_{k}=0
$$

et les coefficients du développement de $P$ dans la base des $P_{k}$ vérifient une relation linéaire non triviale.

Remerciements. Ce travail est issu d'un problème qui a été soumis par Michel Le Bellac à l'un des auteurs (M.Z.) et plusieurs fois rediscuté avec lui. Il doit beaucoup à des discussions avec Pierre Grisvard et Claude Bardos ainsi qu'au travail fait avec Abdelkader Intissar. Nous remercions le Professeur Araki de l'intérêt qu'il lui a porté.

Mme. Laurin a assuré le travail fastidieux que sa qualification n'arrache pas à l'obscurité, celui de la frappe. 


\section{Bibliographie}

1. Ciafaloni, M., Le Bellac, M., Rossi, G.: Reggeon quantum mechanics: a critical discussion. Nucl. Phys. B 130, 388-428 (1977)

2. Grothendieck, A.: Sur certains espaces de fonctions holomorphes. J. Reine Angew. Math. 192, 35-64 (1953)

3. Intissar, A.: Etude d'un opérateur non symétrique intervenant dans la théorie de Regge. Thèse de troisième cycle. Université de Nice 1980

4. Intissar, A.: Sur une propriété spectrale d'un opérateur non symétrique intervenant dans la théorie de Regge. C. R. Acad. Sci. Paris 294, 715 (1982)

5. Intissar, A., Le Bellac, M., Zerner, M.: Properties of the Hamiltonian of reggeon field theory. Phys. Lett. B 1138, 487-489 (1982)

6. Jentzsch, P.: Über Integralgleichungen mit positivem Kern. J. Reine Angew. Math. 141, 235-244 (1912)

7. Kato, T.: Perturbation theory for linear operators. Berlin, Heidelberg, New York: Springer 1966

8. Krein, M.G., Rutman, M.A.: Linear operators leaving invariant a cone in a Banach space. Usp. Mat. Nauk (N.S.) 3, 1 (1948) [Am. Math. Soc. Transl. 26, 3-95 (1960)]

9. Reed, M., Simon, B.: Analysis of operators (Methods of Modern Mathematical Physics IV). New York: Academic Press 1978

10. Schäfer, H.H.: Banach lattices and positive operators. Berlin, Heidelberg, New York: Springer 1974

11. Yosida, K.: Functional analysis, 2ème éd. Berlin, Heidelberg, New York: Springer 1968

Communicated by H. Araki

Reçu le 17 août 1982; accepté le 1 novembre 1983 
